\title{
CONDUCTION PROCESSES IN HIGH VALUE THICK FILM RESISTORS
}

\author{
J. ROBERTSON \\ Electrical Engineering Department, University of Edinjurgh, Edinburgh, EH9 3JL, Scotland
}

(Received June 3, 1977)

\begin{abstract}
This paper describes conduction processes in thick film resistors made from $1 \mathrm{M} \Omega$ /square and $10 \mathrm{M} \Omega /$ square pastes. Conductivity as a function of temperature indicates activation energies in the order of $1 \mathrm{meV}$. At high fields, conductivity is increased. These results are interpreted on the basis of a model where the main resistor characteristics are determined by hopping conduction in a narrow impurity band in the glass phase of the resistor.
\end{abstract}

\section{INTRODUCTION}

An ideal resistor should possess the property of resistance only under all operating conditions. For most applications, thick film resistors are close to this ideal but like all resistor materials, they do show some variation of resistance with operating voltage, temperature, film thickness, electrode separation, electrode material, etc. Most of these effects have been evaluated empirically by thick film paste manufacturers and their results may be incorporated into circuit design rules to ensure that predictable and repeatable hybrid circuits can be produced. At a more fundamental level, accurate description of non-ohmic behaviour provides the key to the identification of basic conduction mechanisms which exist in thick film materials.

High value thick film resistors offer significant advantages in two application areas:

i) High quality, low current circuits. With the advent of cheap FET - input operational amplifiers, many linear circuits can now use resistors in the range $1 \mathrm{M} \Omega$ to $1 \mathrm{G} \Omega$. The main performance criteria are low absolute TCR, good tracking and good long term stability.

ii) High voltage circuits,e.g. reference potential dividers in high voltage power supplies. With $R>$ $100 \mathrm{M} \Omega$, there are few heating problems but voltage coefficient of resistance (VCR) must be minimised.

These applications can be met using resistors with sheet resistance values of $10^{6} \Omega /$ square and above. Linear circuit applications require these resistor materials to have the same low TCR characteristics as lower value resistor pastes but the ability to operate at high fields without significant self heating poses additional problems, the most significant being that VCR provides an ultimate limit to accuracy and linearity.

The work described in this paper arises from the two application areas itemised above and shows how non-ohmic behaviour arises from the conduction mechanism in the resistor film.

\section{CONDUCTION PROCESSES}

Thick film resistor pastes are composed of an active or conducting phase which is finely dispersed through an inert matrix, usually a lead borosilicate glass. The conducting phase is often based on transition metal oxides such as ruthenium oxide, $\mathrm{RuO}_{2}$. If the concentration of the active phase is $>20 \%$, conduction takes place by percolation through channels of conducting material in the glass matrix. The resistor characteristics are essentially those of the active phase only. By varying the composition of the conducting phase, a low net TCR may be achieved over the range 10 to $10^{5} \Omega$ /square.

If pastes of sheet resistance $>10^{6} \Omega /$ square are required, problems arise because it is not possible to maintain a continuous conducting path when the concentration of the active phase falls below about $7 \%{ }^{1}$ The exact figure depends on the morphology of the components ${ }^{2}$ but this sharp discontinuity in conductivity is a general feature of all conductorinsulator mixes. This is shown in Figure 1. When each 


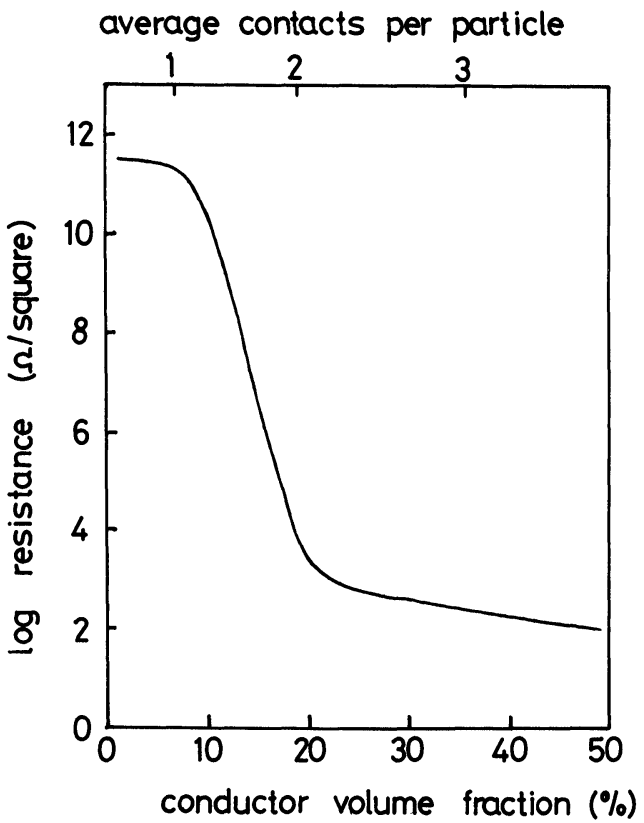

FIGURE 1 Variation of resistivity with composition for a metal-insulator system.

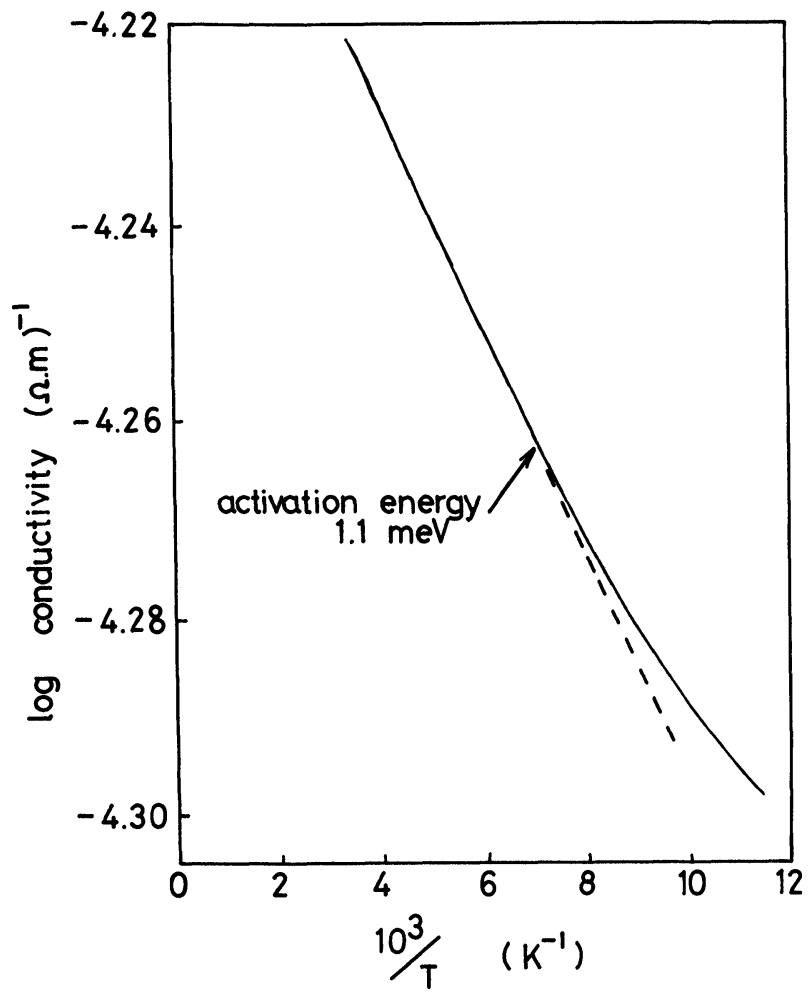

FIGURE 2 Variation of conductivity with temperature for a $10 \mathrm{M} \Omega /$ square resistor. conducting particle has on average less than 2 contacts with other particles, continuous chains no longer exist and resistance rises rapidly. For $\mathrm{RuO}_{2}$ based resistors, this critical concentration is reached at about $1 \mathrm{M} \Omega /$ square $^{3}$ so resistors based on higher sheet resistance values have characteristics which are determined mostly by conduction through the glass between islands of the conducting phase. The more resistivity is increased, the more apparent will be typical semiconductor characteristics such as large negative TCR and VCR. A $10 \mu \mathrm{m}$ thick resistor with $R_{s}=10 \mathrm{M} \Omega /$ square has a resistivity, $\rho=100 \Omega . \mathrm{m}$. By comparison, a semiconducting glass with this resistivity (e.g. $\mathrm{As}_{2} \mathrm{Te}_{3}$ ) has $\mathrm{TCR} \simeq 4 \% \mathrm{~K}^{-1}$. One objective of this paper is to examine how far resistivity may be increased in a thick-film without semiconductor effects becoming predominant.

\section{CONDUCTIVITY MEASUREMENTS}

The variation of conductivity, $\sigma$, with temperature is one of the most widely recognised "fingerprints" of any conduction mechanism. Figure 2 shows an Arrhenius plot of $\log \sigma$ against $T^{-1}$ for a $10 \mathrm{M} \Omega /$ square resistor: Du Pont 9479 . The straight line from room temperature to about $150 \mathrm{~K}$ implies that a valid description of conductivity is given by the equation:

$$
\sigma=\sigma_{1} \exp \left(-\frac{\Delta E}{k T}\right)
$$

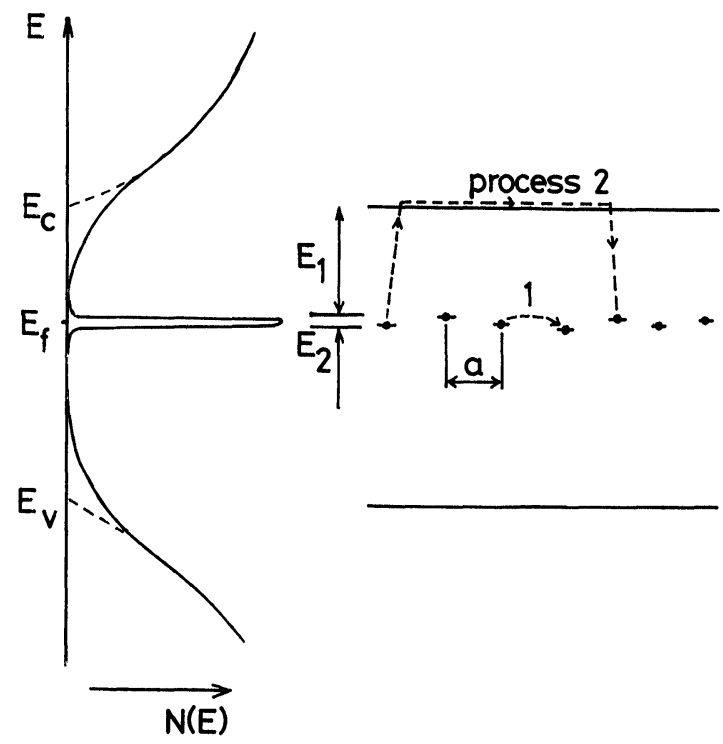

FIGURE 3 Energy band diagram showing possible conduction processes in a doped glass. 
where $\sigma_{1}$ is a constant and $\Delta E$ is an activation energy. In this case, $\Delta E=1.1 \mathrm{meV}$. This is a very small activation energy and it suggests that carrier transport is via states which are all at about the same energy level. The energy band configuration for a doped glass is shown in Figure 3 and it is envisaged that electrons hop between sites at almost constant energy (process 1). Provided the average site separation, $a$, remains small, the hopping process would predominate over thermal ionisation and band conduction (process 2 ).

The model proposed to describe the conduction process is based on the concept that a proportion of the conducting phase in the thick film is randomly dispersed through the glass phase on an atomic scale. It may therefore form a narrow impurity band a few $\mathrm{meV}$ wide within the wide forbidden gap of the glass. ${ }^{4}$ Carrier transport would then occur by a process which involves absorption of lattice energy (phonons) and tunnelling between adjacent impurity sites at almost equal energy levels. The rate of charge transport is therefore relatively insensitive to temperature but very dependent on mean separation between impurity sites and the average field across the sample, i.e. the $\sigma_{1}$ term in Eq. 1 . This is confirmed by measurements carried out on a $1 \mathrm{M} \Omega$ /square resistor film from the same composition family as that shown in Figure 2. Here $\Delta E=0.9 \mathrm{meV}$, indicating a slightly narrower impurity band but the increase in conductivity is almost entirely due to an increase in $\sigma_{1}$.

A reduction in activation energy at low temperature is a common feature of impurity band conduction processes. It may be due to a transition from carrier hopping between nearest neighbours to a variable range hopping process which is even more weakly temperature dependent. An additional factor which may also contribute to the "tail" in Figure 2 is that at low temperatures, phonon energy is reduced and thus carrier activation by phonon absorption is diminished.

\section{HIGH FIELD CONDUCTION}

Deviations from ideal ohmic behaviour are present in all high value thick film resistors when high fields are applied. Since VCR is negative and TCR is also negative for these compositions, some care must be taken to ensure that a reduction in resistance does not arise from self-heating within the resistor.

There are several mechanisms which may give nonohmic behaviour in an amorphous semiconductor.
The principal candidates are: field-assisted hopping $(\sigma \propto \sinh V)$, Poole-Frenkel emission from traps $\left(\sigma \propto \operatorname{expV} \mathrm{V}^{1 / 2}\right)$, high field excitation from traps into the conduction band $(\sigma \propto \exp \mathrm{V})$ and carrier excitation over Schottky barriers either at the electrodes or internally $\left(J \propto \exp \mathrm{V}^{1 / 2}\right)$. In order to identify which of these relationships is applicable, measurements at fields up to $10^{6} \mathrm{~V} \cdot \mathrm{m}^{-1}$ are often necessary. With normal thick film resistor geometries where the electrode separation is $>1 / 2 \mathrm{~mm}$, very high voltages must be used. A more suitable structure for this work is a metal-resistor-metal sandwich. In this case, the bottom electrode was a printed layer of gold-platinum alloy. A $50 \mu \mathrm{m}$ thick resistor layer was produced by depositing 5 successive resistor layers with only a drying stage after each print. The top electrode consisted of a narrow strip of gold which was evaporated over the fired resistor film.

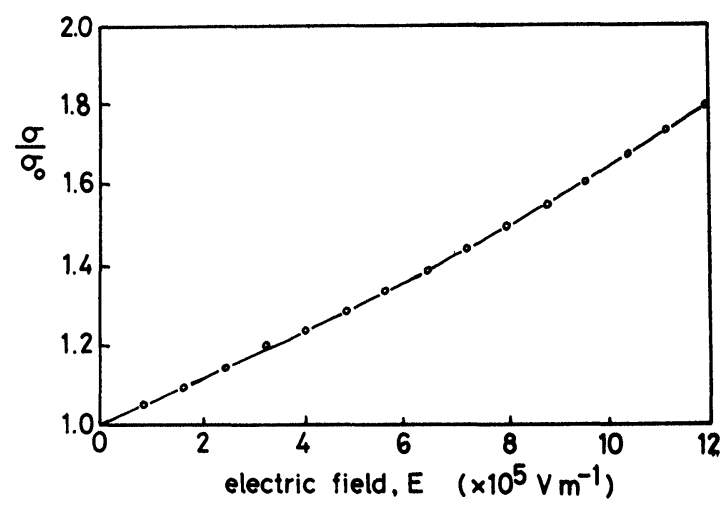

FIGURE 4 Variation of conductivity with field for $10 \mathrm{M} \Omega /$ square resistor.

The resistivity of samples with the sandwich electrode configuration was about half the value derived from the conventional coplanar structure. This is likely to have been caused by diffusion of the bottom contact metal into the resistor film. The effective field within the film is therefore approximately twice its simple $\mathrm{V} / \mathrm{d}$ value. This has been allowed for in the results, but it represents the largest source of error in the deduction of film parameters.

The variation of conductivity with field is shown in Figure 4 for a $10 \mathrm{M} \Omega$ /square resistor. To show the relative influence of field, the conductivity results have been normalised by dividing by $\sigma_{0}$, the appropriate zero field value. All measurements were made under steady state conditions in vacuum and at constant temperature. The results are best 
described by an expression of the form:

$$
\sigma=\sigma_{0} \sinh \left(\mathrm{m}_{1} E\right)
$$

where $m_{1}$ is a constant for any given resistor and $\sigma_{0}$ is the conductivity when field $E=0$. The solid line in Figure 4 represents Eq. 2 for the specified value of $\mathrm{m}_{1}=1.210^{-6} \mathrm{~m} . \mathrm{V}^{-1}$. The results for $1 \mathrm{M} \Omega /$ square resistors are similar but with a different value of $m_{1}$.

This type of high field behaviour is common in transition metal oxide glasses. ${ }^{5}$ The hyperbolic term arises from consideration of the probability of carrier hopping against the field as well as with it. In this case,

$$
\mathrm{m}_{1}=\frac{e}{2 k T} a
$$

where $a$ is the average "jump distance" for a carrier. From Figure 4 the following results may be derived:

\begin{tabular}{rllll} 
& $\mathrm{m}_{1}\left(\mathrm{~m} . \mathrm{V}^{-1}\right)$ & $a(\mathrm{~nm})$ & $N\left(\mathrm{~m}^{-3}\right)$ & $N_{1}(\mathrm{ppm})$ \\
\hline $1 \mathrm{M} \Omega / \mathrm{sq} .9478$ & $7.510^{-7}$ & 12 & $5.10^{23}$ & 50 \\
$10 \mathrm{M} \Omega / \mathrm{sq} .9479$ & $1.210^{-6}$ & 19 & $10^{23}$ & 10 \\
\hline
\end{tabular}

If hopping is between nearest neighbours, then $a$ is a measure of the impurity density in the glass, $N$. Alternatively, the impurity density may be expressed in $\mathrm{ppm}\left(N_{1}\right)$. Although the derived figures for impurity density must be very approximate, they do show that concentration does not scale with resistivity and the atoms of the conducting phase which participate in impurity band conduction in the glass are only a small proportion of the total concentration of active phase in the resistor.

\section{DISCUSSION}

The model which may be used to describe the results of Figures 2 and 4 involves a two-stage conduction process:

i) conduction through short aggregates of the conducting phase.

ii) a series conduction path via a very narrow impurity band in the glass.

The conductivity of the whole film is determined largely by the second process but many resistor characteristics may depend on the uniformity of the dispersion, e.g. high voltage pulsed operation may cause local breakdown if the mix is not homogeneous. This is very similar to the mechanism recently proposed by Ansell. ${ }^{6}$ His results suggested that at
$1 \mathrm{M} \Omega /$ square conduction through glass layers between conducting particles was significant. In this work with a higher resistivity family of resistor materials, conduction through the doped glass phase determines the principal conduction characteristics.

Additional support for this model may be derived from the influence of firing time on resistor properties. With increased time at peak temperatures, high value resistors show some increase in conductivity and decrease in VCR. This may be due to diffusion of more ruthenium ions into the glass phase. Thus, average impurity concentration is increased and the carrier jump distance, $a$, is decreased. If the small aggregates of conducting phase are to be regarded as diffusion sources for the ions which form the impurity band in the glass, this provides an additional case for maintaining close control over particle size and distribution within the resistor film.

Perhaps the most important extension of this work concerns basic limits which may be placed on the maximum sheet resistance which can be achieved without being accompanied by large TCR and VCR values. As the concentration of conducting phase is reduced in any resistor composition, the overlap integral between sites in the impurity band is decreased. There is then likely to be an abrupt transition from band-type conduction via delocalised states to hopping between isolated traps. The latter process gives the high activation energies which are observed in most semiconducting glasses (cf. process 2, Figure 3).

It is more difficult to predict the value of sheet resistance at which this change in conduction mechanism will occur. However, there are two reasons for thinking that $10 \mathrm{M} \Omega /$ square may be near the limit:

i) The average jump distance, $a=19 \mathrm{~nm}$ and tunnelling decreases very rapidly for distances above $10 \mathrm{~nm}^{7}$

ii) If the impurity density, $N$, is confined to a band $5 \mathrm{meV}$ wide, it represents a density of states, $N_{f}=2.10^{5} \mathrm{~m}^{-3} \mathrm{eV}^{-1}$ at the Fermi level (in the impurity band). This is approximately the value for the density of states at the bottom of the conduction band in a semiconductor. Any reduction below this value for $N_{f}$ implies that a conduction band state no longer exists and it will be superceded by a typical high TCR, high VCR semiconductor conduction process.

The best long-term options for production of high value thick film resistors may therefore lie in producing long tracks within a small area rathern than developing ever-higher values of sheet resistance. 


\section{REFERENCES}

1. S. M. Aharoni, J. App. Phys. , 43, 5, 1972, 2463-2465.

2. A. Malliaris and D. T. Turner, J. App. Phys. , 42, 2, 1971, 614-618.

3. P. F. Carcia et al., Proc. Internepcon, Brighton, 1974.
4. N. F. Mott and E. A. Davis, Electronic Processes in NonCrystalline Materials, Oxford U.P., 1971.

5. I. G. Austin and M. Sayer, J. Phys. C., 7, 1974, 905-914.

6. M. P. Ansell, Electrocomponent Sci. and Tech., 3, 1976, $131-151$.

7. J. Simmons, J. App. Phys., 34, 1963, 1793-1803. 

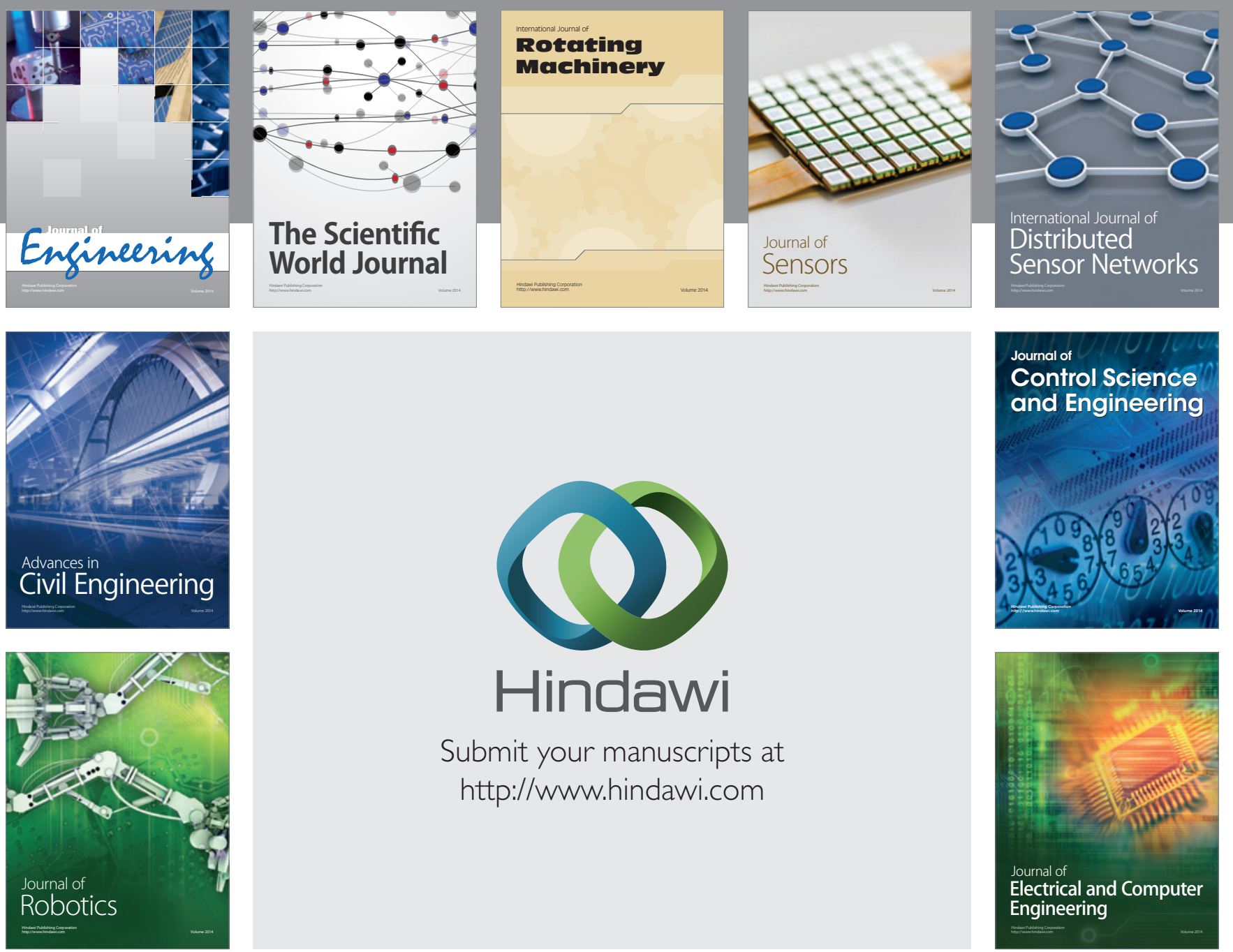

Submit your manuscripts at

http://www.hindawi.com
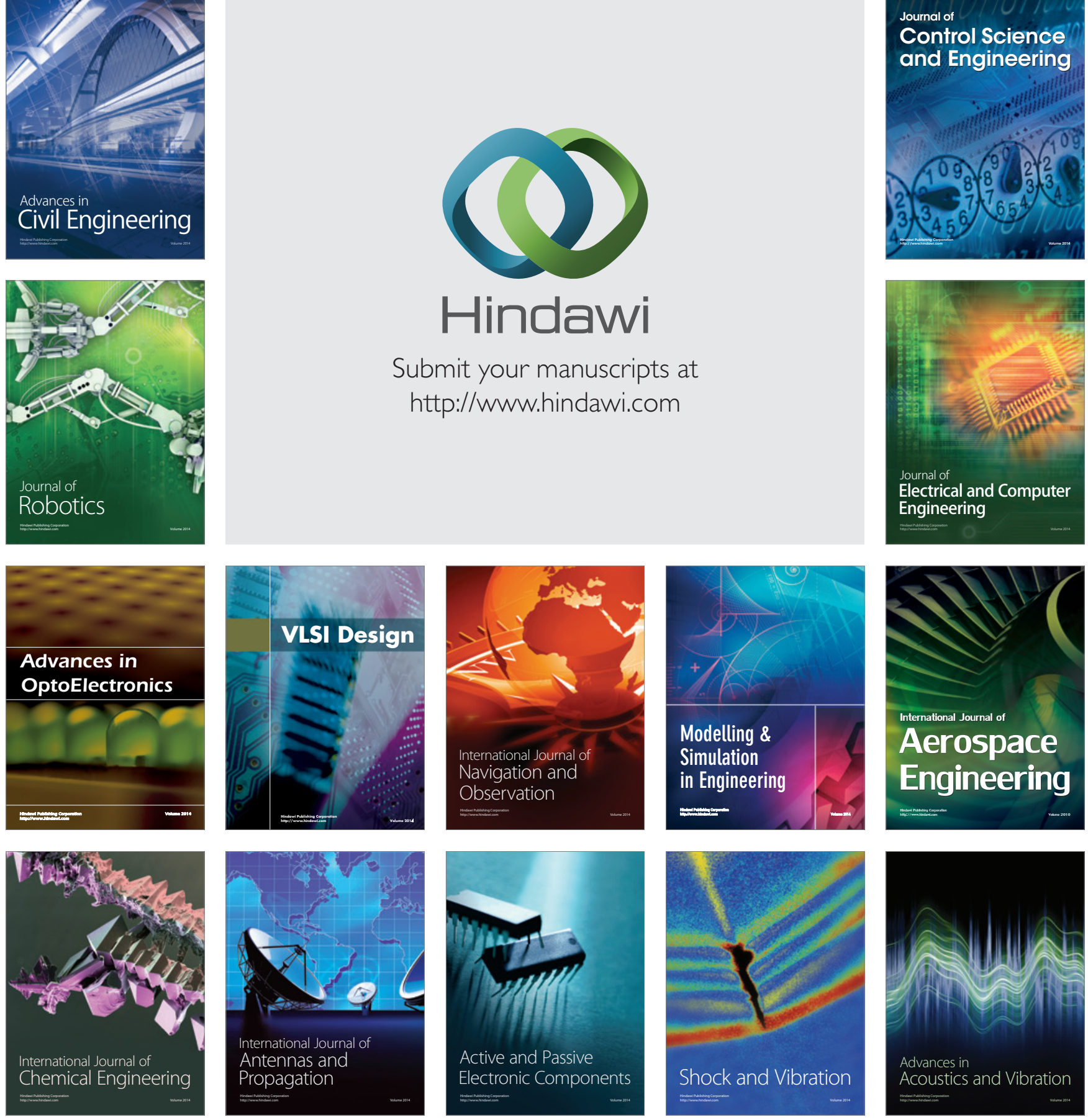